\title{
Regulation of endogenous brakes to kidney fibrosis: turning the view upside down
}

\author{
Jonathan A. Lindquist ${ }^{1}$ • Alexandra Schneider ${ }^{1} \cdot$ Peter R. Mertens $^{1}$
}

Published online: 17 May 2017

(C) Springer-Verlag Berlin Heidelberg 2017

It is common knowledge that kidney injury mostly results in a self-perpetuating and sustaining disease with poor outcome. The nephrology community is notorious for the lack of efficient interventions to combat this relentless process characterized by kidney fibrosis and diminished excretion of nitrogenous wastes. At this point, one may change the point of view and ask the question whether the kidney itself is the answer for this failure of treatment options and the epicentrum of dysbalance. The study by Yu et al. [1] provides compelling evidence that this altered perspective holds true and provides novel answers. Impaired kidney function goes along with rising levels of uremic toxins, of which there are more than 120 substances that mediate adverse effects on cognition and organ functions, such as digestion in the bowels and muscle weakness, appetite, and bone metabolism. Two uremic toxins have been analyzed in detail, indoxyl sulfate as well as pcresyl sulfate [2]. Both are already elevated at early stages of kidney impairment, importantly, the serum levels of indoxyl sulfate are up to fivefold elevated with chronic kidney disease and highly correlate with tubulointerstitial kidney fibrosis $(r>0.9)$. This is the starting point for Yu et al. [1] to link uremia toxins with kidney fibrosis and to dissect the underlying molecular pathways. Given such a strong association, it is

Comment on: Yanlin Yu, Xu Guan, Ling Nie, Yong Liu, Ting He, Jiachuan Xiong, Xinli Xu, Yan Li, Ke Yang, Yiqin Wang, Yunjian Huang, Bing Feng, Jingbo Zhang, Jinghong Zhao. DNA hypermethylation of sFRP5 contributes to indoxyl sulfate-induced renal fibrosis

Peter R. Mertens

peter.mertens@med.ovgu.de

1 Clinic of Nephrology and Hypertension, Diabetes and Endocrinology, Otto-von Guericke University, Leipziger Str. 44, 39120 Magdeburg, Germany astonishing how many nodes are involved in this process. A brief summary includes NADPH oxidase, reactive oxygen species, mitogen-activated protein kinase ERK1/2, DNA methyltransferases, secreted frizzled-related proteins (sFRP), klotho, $\mathrm{Wnt} / \beta$-catenin signaling, $\alpha$-smooth-muscle actin, collagens, and matrix metalloproteinase-7. Confused by this long list? Most players are notorious for their participation in organ fibrosis (overview in Fig. 1). Yu et al. elucidate that indoxyl sulfate application induces epigenetic phenomena, which involve the methylation of $\mathrm{CpG}$ islands within target genes, such as klotho and secreted frizzled-related protein-5. These methylation steps are performed by DNA methyltransferases (DNMTs) that are upregulated in tubular cells due to oxidative stress and MAP kinase signaling. Among the family of secreted frizzled-related proteins denoted 1 through 5 , only one is upregulated following indoxyl sulfate application, sFRP-5. This protein acts extracellularly and sequesters Wnt-5a, thereby preventing its binding to frizzled receptors and inducing $\beta$ catenin signaling (see Fig. 2). In other words, less sFRP-5 results in increased $\mathrm{Wnt} / \beta$-catenin signaling. Other studies revealed a similar downregulation of sFRP-4 in unilateral ureteral obstruction [3]. An analogous inhibitory activity has been described for the secreted klotho protein. For the Wnt/ $\beta$-catenin signaling, ample evidence and mechanisms are known on how this promotes organ fibrosis and destruction of tissue architecture, e.g., in the kidney with tubulointerstitial fibrosis [4].

To confirm all the described steps and determine the relative contribution of the proponents, Yu et al. [1] applied different interventions with immortalized tubular cell lines in murine models of kidney disease following uninephrectomy and intraperitoneal application of indoxyl sulfate. As schematically summarized in Fig. 1, the interventions encompassed the ROS scavenger N-acetylcysteine (NAC), MEK inhibition (U0126), DNA methyltransferase inhibitor decitabine (5-aza- 


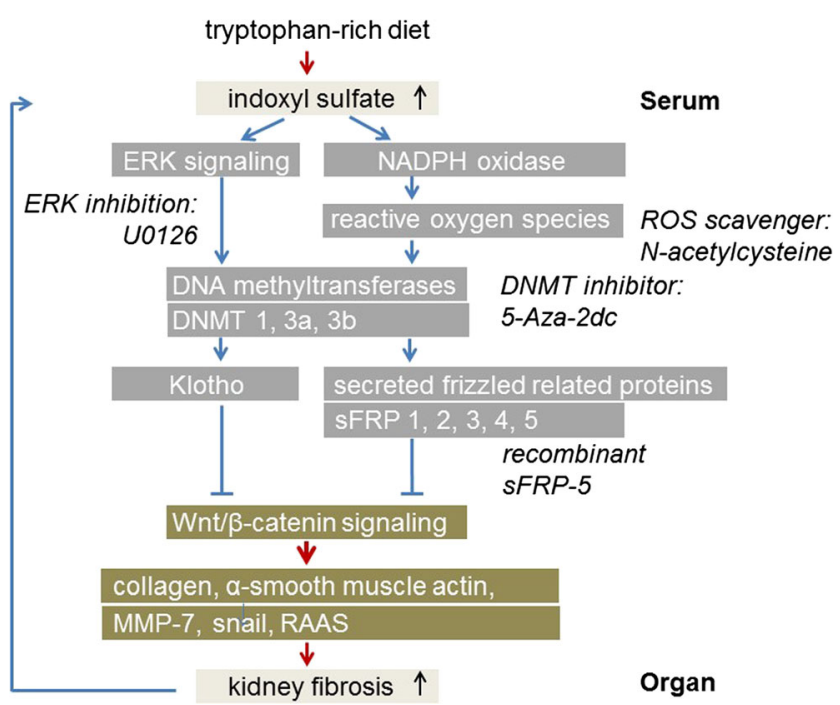

Fig. 1 Interacting nodes from the uremic toxin indoxyl sulfate to kidney fibrosis. Dietary tryptophan is broken down by commensal bacteria within the gut, leading to the production of indoxyl sulfate. Within the plasma, this protein-bound toxin eludes excretion and serum levels increase over time. Indoxyl sulfate activates signaling cascades, e.g., MAP kinases and ROS, thereby inducing the expression of DNA methyltransferases. These, in turn, methylate $\mathrm{CpG}$ islands found within the genes for klotho and secreted frizzled-related protein (sFRP), thereby suppressing their expression. Reduced levels of extracellular klotho and sFRP lead to an enhanced activation of the Wnt/ $\beta$-catenin signaling pathway, which induces a number of pro-fibrotic genes, e.g., collagen I, $\alpha$-smooth muscle actin, matrix metalloproteinase-7, snail, RAAS; all of which contribute to kidney fibrosis. Fibrosis diminishes kidney function, resulting in increased levels of indoxyl sulfate within the serum, which aggravates the situation by increasing inflammation and reinforcing organ fibrosis. Uninterrupted, this self-perpetuating cycle will ultimately result in organ failure

2'-deoxycytidine), and intraperitoneal application of recombinant sFRP-5 protein to sequester Wnt ligands. All these

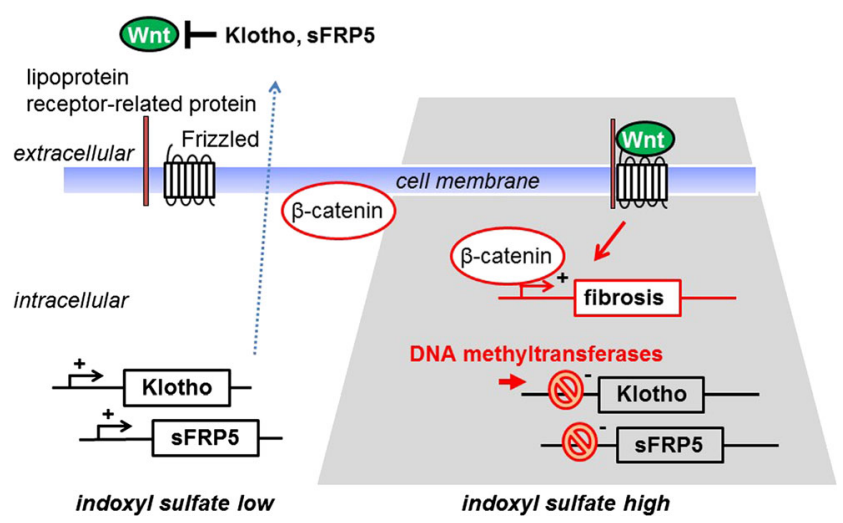

Fig. 2 Inhibition of $\mathrm{Wnt} / \beta$-catenin signaling by klotho and secreted frizzled-related protein. In healthy individuals, klotho and fizzledrelated proteins are secreted from the cell to prevent Wnt signaling (induced via binding to its receptor frizzled and the co-receptor lipoprotein receptor-related protein). In CKD patients, the accumulation of indoxyl sulfate in serum induces the expression of DNA methyltransferases (DNMTs) within cells, which methylate $\mathrm{CpG}$ islands within the klotho and sFRP genes, thereby turning the genes OFF. This allows Wnt to bind its receptor(s) and induce the expression of fibrosisrelated genes, thereby causing organ fibrosis interventions abrogated $\mathrm{Wnt} / \beta$-catenin signaling, the last two interventions proved to protect from kidney fibrosis mediated by indoxyl sulfate application in murine kidney disease models.

The findings are convincing and seemingly make an intervention to kidney fibrosis easy. What is the missing link for translation into clinic?

Some applied interventions seem to be easy to perform, however, have not been successful. N-acetylcysteine (NAC) has been applied to prevent contrast media toxicity, however, only for a few days. For patients with chronic kidney disease, short-term treatment with NAC $(4 \times 1200 \mathrm{mg} / 12 \mathrm{~h})$ showed no improvement in kidney function [5]. Thus, a long-term trial would be required to answer the question whether this compound may be suitable for a renaissance. Highly selective MAPK inhibitors (trametinib, selumetinib) are in clinical trials at different stages, mostly for melanoma and thyroid cancer. Common side-effects reported for these substances include rashes and diarrhea. As ERK signaling is implicated in a number of pro-fibrotic signaling pathways, these drugs may also hold promise for kidney diseases; however, until now no clinical trials have been registered for kidney diseases at either the EU Clinical Trials Register or ClinicalTrials.gov. DNA methyltransferase inhibitor decitabine is an FDA-approved treatment since 2006 for myelodysplastic syndrome and mostly applied to elderly patients. However, for the kidney, testing of its efficacy has so far been limited to prospective studies on renal cell carcinoma.

Application of recombinant proteins, such as klotho and sFRP-5 seems feasible. The chosen dosages in the animal experiments $(10 \mu \mathrm{g} / \mathrm{kg} /$ day $)$ are reasonable; however, extensive analyses will be required on the pharmacokinetics, pharmacodynamics, as well as immunogenicity. The data so far suggest that both proteins are effective treatments for reducing renal fibrosis [1,6]. Although a number of clinical trials are investigating klotho as a potential biomarker as well as its role in regulating phosphate and vitamin D metabolism, no study has been registered to investigate its anti-fibrotic activity.

The beneficial outcome following application of these distinct compounds in experimental models of kidney disease may make patients and caring physicians optimistic; however, kidney diseases do not progress rapidly and most require patience on both sides before one may assess the outcome.

One intriguing option to interfere in the kidney disease progress resides in the elimination of protein-bound uremia toxins such as indoxyl sulfate and p-cresyl sulfate. Such an intervention has been tested in clinical trials with the charcoalbased drug AST-120; however, the amount one needs to consume $(30 \times 300 \mathrm{mg}$ capsules/day) are a challenge and impairs patient compliance, with only $\sim 40 \%$ of each group completing the study [7]. Alternate strategies would be to reduce the generation of uremia toxins. Dietary protein is broken down into amino acids within the gut. Of these, the aromatic amino 
acids, tryptophan, tyrosine, and phenylalanine are converted to indole or p-cresol by commensal bacteria. These compounds are then absorbed into the blood/plasma and, in the liver, conjugated with sulfuric acid to form indoxyl sulfate and p-cresyl sulfate, which then bind to albumin and circulate as protein-bound toxins. Putatively, the intake of these amino acids may be restricted, which contradicts nutritional supplementation recommendations that advertise and market these amino acids as safe natural health products. Numerous alleged benefits have been described for tryptophan, presenting it as a remedy against mood disorders and natural sleeping aid. Given the accumulation of indoxyl sulfate following increased tryptophan ingestion and along this line expected additional kidney fibrosis, a closer look should be taken on a patient's diet and nutrient supplements. This is of particular importance, as circulating uremic toxins increase permeability within the gut, thereby enhancing inflammation and inducing dysbiosis, a disturbance in the normal gut microbiota, which has been implicated in a number of diseases including obesity, diabetes, inflammatory bowel, cardiovascular, and kidney diseases. Alterations in the intestinal microbial flora have been identified in patients with end-stage renal disease and have been induced in animal models of uremia within 8 weeks. Application of probiotics to correct the dysbiosis may prove beneficial [2].

Finally, epigenetics strongly affects organ fibrosis. There are some known drugs harboring epigenetic "side-effects" and that inadvertently have the potential to halt kidney disease. One such example is hydralazine, a compound known for its blood pressure lowering effects [8]. Thus, epigenetics holds the promise that this long known drug experiences a renaissance. Keep your eyes open. Another important message from the study by $\mathrm{Yu}$ et al. [1] relates to endogenous anti-fibrotic mechanisms. Klotho and secreted frizzled-related proteins function as Wnt antagonists, thus acting as endogenous brakes to combat organ fibrosis. Suppressing the expression of these proteins enhances organ fibrosis. This is not only true for the kidney, but similar mechanisms exist also in the liver and skin [9, 10].

Finally, the idea of a serum marker for uremia, other than urea and creatinine, with relevance for organ fibrosis is compelling. One may envision that treatment adjustments may be guided according to the serum levels of indoxyl sulfate and pcresyl sulfate, as an example. However, these are at the moment only quantified by fluorescence liquid chromatography.
As a consequence, risk for organ fibrosis would be quantifiable, similar to autoimmune disorders (autoantibody titers) or cancer diseases (tumor markers) and treatment options may be adjustable. What a nice visionary aspect, for patients and caring doctors alike - a personalized fibrosis medicine 1.0.

Acknowledgements Work in the authors' laboratory is funded by DFG grants LI-1031/4-1 to JAL, and ME-1365/7-2, ME-1365/9-1, and SFB854 TP-A01 to PRM.

\section{References}

1. Yu Y, Guan X, Nie L, Liu Y, He T, Xiong J, Xu X, Li Y, Yang K, Wang Y et al (2017) DNA hypermethylation of sFRP5 contributes to indoxyl sulfate-induced renal fibrosis. J Mol Med (Berl). doi:10. 1007/s00109-017-1538-0

2. Rossi M, Johnson DW, Morrison M, Pascoe EM, Coombes JS, Forbes JM, Szeto CC, McWhinney BC, Ungerer JP, Campbell KL (2016) Synbiotics easing renal failure by improving gut microbiology (SYNERGY): a randomized trial. Clin J Am Soc Nephrol 11(2):223-231

3. Surendran K, Schiavi S, Hruska KA (2005) Wnt-dependent betacatenin signaling is activated after unilateral ureteral obstruction, and recombinant secreted frizzled-related protein 4 alters the progression of renal fibrosis. J Am Soc Nephrol 16(8):2373-2384

4. He W, Dai C, Li Y, Zeng G, Monga SP, Liu Y (2009) Wnt/betacatenin signaling promotes renal interstitial fibrosis. J Am Soc Nephrol 20(4):765-776

5. Moist L, Sontrop JM, Gallo K, Mainra R, Cutler M, Freeman D, House AA (2010) Effect of N-acetylcysteine on serum creatinine and kidney function: results of a randomized controlled trial. Am J Kidney Dis 56(4):643-650

6. Hu MC, Shi M, Gillings N, Flores B, Takahashi M, Kuro-O M, Moe OW (2017) Recombinant alpha-Klotho may be prophylactic and therapeutic for acute to chronic kidney disease progression and uremic cardiomyopathy. Kidney Int 91(5):1104-1114

7. Schulman G, Berl T, Beck GJ, Remuzzi G, Ritz E, Arita K, Kato A, Shimizu M (2015) Randomized Placebo-Controlled EPPIC Trials of AST-120 in CKD. J Am Soc Nephrol 26(7):1732-1746

8. Tampe B, Steinle U, Tampe D, Carstens JL, Korsten P, Zeisberg EM, Müller GA, Kalluri R, Zeisberg M (2017) Low-dose hydralazine prevents fibrosis in a murine model of acute kidney injury-tochronic kidney disease progression. Kidney Int 91(1):157-176

9. Bai J, Liu Z, Xu Z, Ke F, Zhang L, Zhu H, Lou F, Wang H, Fei Y, Shi YL et al (2015) Epigenetic downregulation of SFRP4 contributes to epidermal hyperplasia in psoriasis. J Immunol 194(9):41854198

10. Miao CG, Yang YY, He X, Huang C, Huang Y, Zhang L, Lv XW, Jin Y, Li J (2013) Wnt signaling in liver fibrosis: progress, challenges and potential directions. Biochimie 95(12):2326-2335 